\title{
Basal-like breast cancer with low TGF $\beta$ and high TNFa pathway activity is rich in activated memory CD4 T cells and has a good prognosis
}

\author{
Dingxie Liu ${ }^{1 凶}$, Jaydutt Vadgama ${ }^{2 \bowtie}$ and Yong $\mathrm{Wu}^{2 \bowtie}$ \\ 1. Bluewater Biotech LLC, New Providence, NJ, USA. \\ 2. Division of Cancer Research and Training, Department of Internal Medicine, Charles Drew University of Medicine and Science, David Geffen UCLA School \\ of Medicine and UCLA Jonsson Comprehensive Cancer Center, Los Angeles, CA 90095, USA. \\ $\bowtie$ Corresponding authors: Dingxie Liu, Ph.D. Bluewater Biotech LLC, PO Box 1010, New Providence, NJ 07974, USA. E-mail: dliu@bluewater-biotech.com; \\ Jaydutt V. Vadgama, Division of Cancer Research and Training, Department of Internal Medicine, Charles R. Drew University of Medicine and Science, David \\ Geffen UCLA School of Medicine, and UCLA Jonsson Comprehensive Cancer Center, 1748 E. 118th Street, Los Angeles, CA, 90059, USA. Phone: 323-563-9397; \\ Fax: 323-563-4889; E-mail: jayvadgama@cdrewu.edu. Yong Wu, Ph.D. Division of Cancer Research and Training, Department of Internal Medicine, Charles R. \\ Drew University of Medicine and Science, David Geffen UCLA School of Medicine, and UCLA Jonsson Comprehensive Cancer Center, 1748 E. 118th Street, Los \\ Angeles, CA, 90059, USA. E-mail: yongwu@cdrewu.edu.
}

(C) The author(s). This is an open access article distributed under the terms of the Creative Commons Attribution License (https://creativecommons.org/licenses/by/4.0/). See http://ivyspring.com/terms for full terms and conditions.

Received: 2020.11.24; Accepted: 2020.12.29; Published: 2021.01.30

\begin{abstract}
Basal-like breast cancer (BLBC) is a type of high-grade invasive breast cancer with high risk of recurrence, metastases, and poor survival. Immune activation in BLBC is a key factor that influences both cancer progression and therapeutic response, although its molecular mechanisms are not well clarified. In this study, we examined five cancer immunity-related pathways (IFNa, IFNy, STAT3, TGF $\beta$ and TNFa) in four large independent breast cancer cohorts $(n=6,381)$ and their associations with the prognosis of breast cancer subtypes. Activities of the 5 pathways were calculated based on corresponding pathway signatures and associations between pathways and clinical outcomes were examined by survival analysis. Among the five PAM50-based subtypes, BLBC had the highest IFNa, IFNy, TNFa pathway activities, and the lowest TGF $\beta$ activity. The IFNa, IFNy, TNFa pathway activities were negatively correlated with BLBC recurrence. In contrast, positive association and no association with BLBC recurrence were observed for TGF $\beta$ and STAT3 pathways, respectively. TNFa/TGF $\beta$ pathway combination improved the prediction of recurrence and chemotherapy response of BLBCs. Immune cell subset analysis in BLBC showed that M0, $M 1$ and M2 macrophage levels were associated with either TNFa or TGF $\beta$ pathways, whereas the level of activated memory CD4 T cells were associated with both pathways. Moreover, this T cell subset was most abundant in BLBCs with low TGF $\beta$ and high TNFa pathway activities. These results suggested that cooperation of TNFa and TGF $\beta$ signaling may be involved in the regulation of memory $T$ cells and anti-cancer immunity in BLBCs. Our data also demonstrate that TNFa/TGF $\beta$ pathway combination may represent a better biomarker for BLBC prognosis and clinical management.
\end{abstract}

Key words: Basal-like breast cancer, immunity-related pathways, chemotherapy, prognosis

\section{Introduction}

Breast cancer is the most common malignant tumor in women and a heterogeneous disease that has been demonstrated to be divided into five major biologically distinct intrinsic subtypes based on gene expression profiling, i.e. luminal A, luminal B, human epidermal growth factor receptor-2 (HER2) overexpressing, basal-like, and normal-like [1-3]. Of the five intrinsic subtypes, basal-like breast cancer
(BLBC) is of particular concern since it does not express estrogen receptor (ER), progesterone receptor (PR), and HER2, and hence does not benefit from anti-estrogenic therapy or trastuzumab [4]. BLBC is likely to be a high-grade invasive tumor with active mitosis and is related to younger patient age $[5,6]$.

Recently, whole genome analysis has been supplemented to the equipment library of 
experimental techniques, which provides a new molecular classification for breast cancer and promotes the development of many prognostic multigene analyses [7-13]. The initial gene expression profile analysis showed that BLBCs and HER2 overexpression subtypes are associated with exceptionally poor prognosis [14-16]. In contrast, patients with Luminal A cancers have a good prognosis [2, 3]. Nevertheless, after careful examination, these studies further indicate that the prognosis of BLBC patients is highly time-dependent. Some patients with BLBCs have a particularly low survival rate in the first 3-5 years after diagnosis, nonetheless for others, their mortality rate decreases, so the survival rate of these patients is better than that of patients with luminal (ER1) tumors at 10 years after diagnosis [17-20]. This indicates that patients with BLBCs can be divided into two clinically distinct groups: those who are likely to relapse and die of their disease within the first three to five years of diagnosis, and those who are expected to show good long-term survival. Therefore, the indexes that can be used to accurately predict the prognosis of this kind of patients are particularly important and have clinical significance.

Although there are a variety of multi-gene signatures that can predict the prognosis of patients with breast cancer, their prognostic value seems to come mainly from their ability to measure the expression of proliferation-related genes [21, 22]. Since BLBC is usually highly proliferative, the existing prognostic signatures fail to identify BLBC subsets with a favorable prognosis [23]. Some recent studies have focused on determining multi-gene predictors for the prognosis of triple-negative (ER-, PR-, HER2-) and hormone receptor negative breast cancers [22-27]. Nevertheless, a reliable method to distinguish between good and poor prognosis of BLBC has not been developed. For this purpose, we have begun to optimize such a method, and here we report the identification of an immunological pathway signature combination, which is related to the prognosis of patients with BLBCs.

Although genetic/epigenetic alterations are key pathogenic events, the immunological signaling plays an important role in promoting progression and metastasis of BLBCs. Previously, we examined multiple cancer-related pathway signatures in colon and breast cancers [28-30]. Five of these signatures, including IFNa, IFN $\gamma$, STAT3, TGF $\beta$ and TNFa pathway signatures, are cancer immunity-related. In this study, we analyzed the associations of these signatures with the prognosis of PAM50-based intrinsic subtypes of breast cancers, with an emphasis on BLBC.

\section{Methods}

\section{Microarray datasets of breast cancer}

In our previous study [29-31], we tested 42 Affymetrix U133 microarray datasets, which were again used to examine the correlation between immunological pathway signatures and basal-like breast cancer. In the 42 microarray datasets, 15 datasets contain patients' neoadjuvant response information, and 30 data sets contain cancer recurrence information (3 data sets contain both information). We described in detail the normalization of the raw array data, the elimination of batch effects, and the clinical characteristics of patients in these datasets in our previous work [29, 31].

We previously merged the 42 microarray datasets into 4 cohorts [29-31]. Cohort 1 was used here to examine the association of pathway signatures with neoadjuvant response of $\mathrm{BC}$ because it was merged from 15 datasets with only patient neoadjuvant response information (i.e. MDA133, GSE16446, GSE18728, GSE18864, GSE20194， GSE20271, GSE25055, GSE25065, GSE26639, GSE32646, GSE37946, GSE41998, GSE42822, GSE50948, and GSE66305). The other 3 cohorts were used to examine the associations of pathway signatures with BC relapse. Cohort 2 contains 16 datasets (GSE11121, GSE12276, GSE2034, GSE17705, GSE2603, GSE20685, GSE2990, GSE26971, GSE3494, GSE45255, GSE58812, GSE6532, GSE65194, GSE7390, GSE9195, GSE88770) in which metastasis date is available. Cohort 3 comprises 8 datasets (GSE12093, GSE20711, GSE21653, GSE31519, GSE42568, GSE1456, GSE7378, GSE71258) in which only relapse date information is available. The remaining 6 datasets with RFS or DMFS information (GSE16391, GSE16446, GSE17907, GSE19615, GSE25055, and GSE25065) were merged as the Cohort 4 because the follow-up time of these datasets (about 5 years) was much shorter than that of cohorts 2 and 3 . The batch effect in the four merged cohorts was eliminated using the R Combat function, as we showed previously [29]. The disease-free survival time in cohort 2 and cohort 3 was censored at 8 years, while it was censored at 5 years in cohort 4 .

\section{Pathway signature generation and pathway activity prediction using BinReg}

Using the BinReg approach to generate pathway signatures and predict pathway activities of individual samples has been described in detail before $[32,33]$. Briefly, the gene expression patterns of two groups of samples (with one pathway being 'on' and 'off' in the two groups respectively) are analyzed to identify the pathway-specific informative genes 
(signature genes). Principal components are then used to compute weights for each of signature genes, such that the weighted average of expression levels show a clear ability to distinguish the pathway "on" and "off" groups. By applying binary regression on the principal components to the gene expression dataset of a test sample, a probability score of pathway activity for that sample will be produced.

The signatures for 11 pathways analyzed in this study, except for BRAF/MEK and AR pathways, were previously reported by Gatza et al [32, 33]. According to the authors' suggestion, MAS5.0 normalized gene expression data was used to predict the activity of p53, STAT3, and TNFa pathways, while RMA normalized data was used for Wnt/ $\beta$-catenin (BCAT), EGFR, IFNa, IFN $\gamma$, PI3K and TGF $\beta$ pathways. AR signature was generated in our previous study [29], while BRAF/MEK was generated in the present study. Both of these two signatures were based on RMA-normalized expression data.

IFNa, IFN $\gamma$, TGF $\beta$ and TNFa pathway signatures were generated and validated using gene expression data from cells treated with or without IFNa, IFN $\gamma$, TGF $\beta$ and TNFa, respectively [33]. We previously generated a gene signature for mutant BRAF signaling [34]. Since BRAF mutation is rare in breast cancer, here we generated a BRAF/MEK signature based on the cells treated with mock or MEK-specific inhibitor (Supplementary Fig. S1). The conditions for the BRAF/MEK pathway signatures are listed in Supplementary Table S1.

\section{Score calculation of two pathway signature combination}

The pathway signature scores were first Z-sore transformed. The scores for combination of TGF $\beta$ with IFNa, IFN $\gamma$, and TNFa were calculated by subtracting the TGF $\beta$ signature score from IFNa, IFN $\gamma$, and TNFa signature scores respectively (e.g., score of $\mathrm{TNFa} / \mathrm{TGF} \beta$ combination $=\mathrm{TNFa}$ signature score - TGF $\beta$ signature score).

\section{Prediction of intrinsic subtypes of breast cancer}

The intrinsic subtypes luminal A (LumA), luminal B (LumB), HER2-enriched, normal-like and basal-like were predicted by using the PAM50 centroids in the Genefu $\mathrm{R}$ package [35]. RMA normalized gene expression data was used.

\section{Intratumoral immune cell composition analysis}

CIBERSORT (https://cibersort.stanford.edu/) was used to calculate immune cell subtype fractions based on the gene expression profile of each breast cancer sample [36]. Gene signatures (LM22) for 22 sorted immune cell subsets were used in this study. Quantile normalization was used as recommended to remove confounding effects. We chose LM22 here because the prediction power of these signatures for corresponding immune cell subsets were well experimentally validated [36].

\section{Statistics}

Statistics was performed by using $\mathrm{R}$ packages including Metafor, Survival, and Survminer. Odds-ratios (OR) for the associations of neoadjuvant response with risk scores were calculated using logistic regression. The association between disease-free survival and risk scores was analyzed using Kaplan-Meier survival curves with log-rank test and cox proportional hazards regression. Disease free survival (DFS) was defined as the time from surgery to the first confirmed relapse or metastasis. In this study distant metastasis-free survival (DMFS) was preferred to be used in survival analysis, while when the data is not available, relapse-free survival (RFS) data was used. The overall hazard ratio (HR) of a variable of interest was calculated using a random-effects model. The significance of the overall effects across multiple datasets was estimated by $\mathrm{Z}$ test.

All statistical analyses were two-sided and considered significant when $p<0.05$.

\section{Results}

\section{IFNa, IFNy and TNFa pathways are up-regulated while TGF $\beta$ pathway is down-regulated in BLBC}

Activities of the IFNa, IFN $\gamma$, TNFa TGF $\beta$ and STAT3 pathways, which were predicted with corresponding signatures, were compared among five PAM50-based BC subtypes. The first four pathways showed different expression pattern among different BC subtypes (Fig. 1). The IFNa and IFNY pathways showed higher levels in HER2-enriched BCs and BLBCs but lower levels in luminal A and B BCs. The TNFa pathway activity increased gradually from luminal A, luminal B, Normal-like, HER2-enriched and basal-like subtypes. Conversely, the TGF $\beta$ pathway exhibited an opposite activity pattern with TNFa pathway. Overall, the pathway activities of IFNa, IFN $\gamma$, TNFa were the highest, while that of TGF $\beta$ was the lowest in BLBC. No significant difference of the STAT3 pathway activity was observed among the five PAM50 subtypes (Fig. 1). 


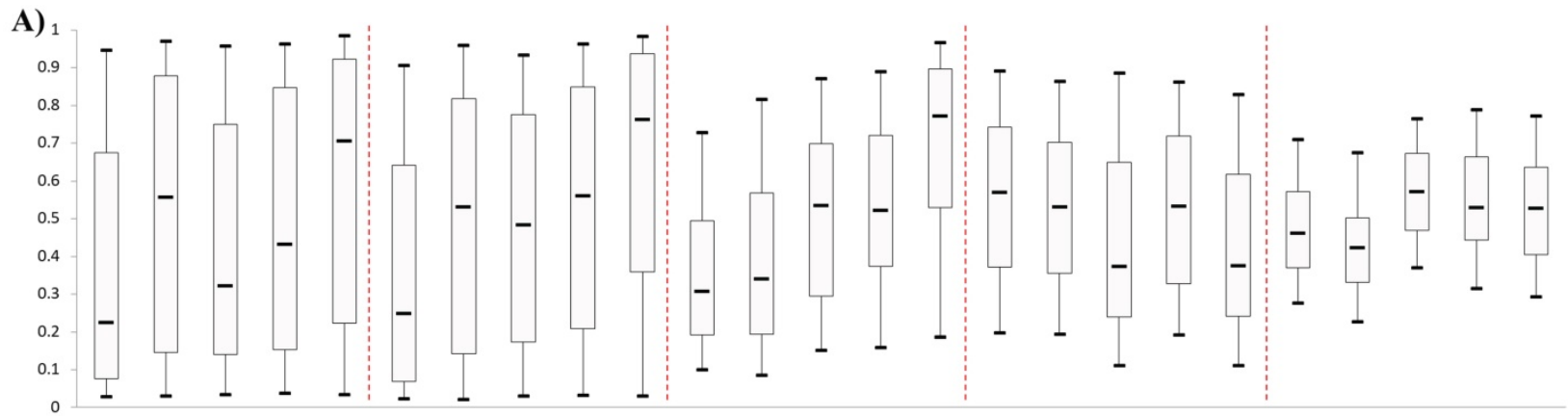

Subtype LumA LumB Normal Her2 Basal LumA LumB Normal Her2 Basal LumA LumB Normal Her2 Basal LumA LumB Normal Her2 Basal LumA LumB Normal Her2 Basal

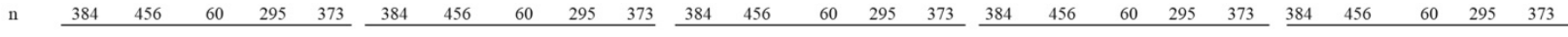
$\begin{array}{llll}\text { IFN } \alpha \text { pathway } & \text { IFN } \gamma \text { pathway } & \text { TNF } \alpha \text { pathway } & \text { pathway }\end{array}$
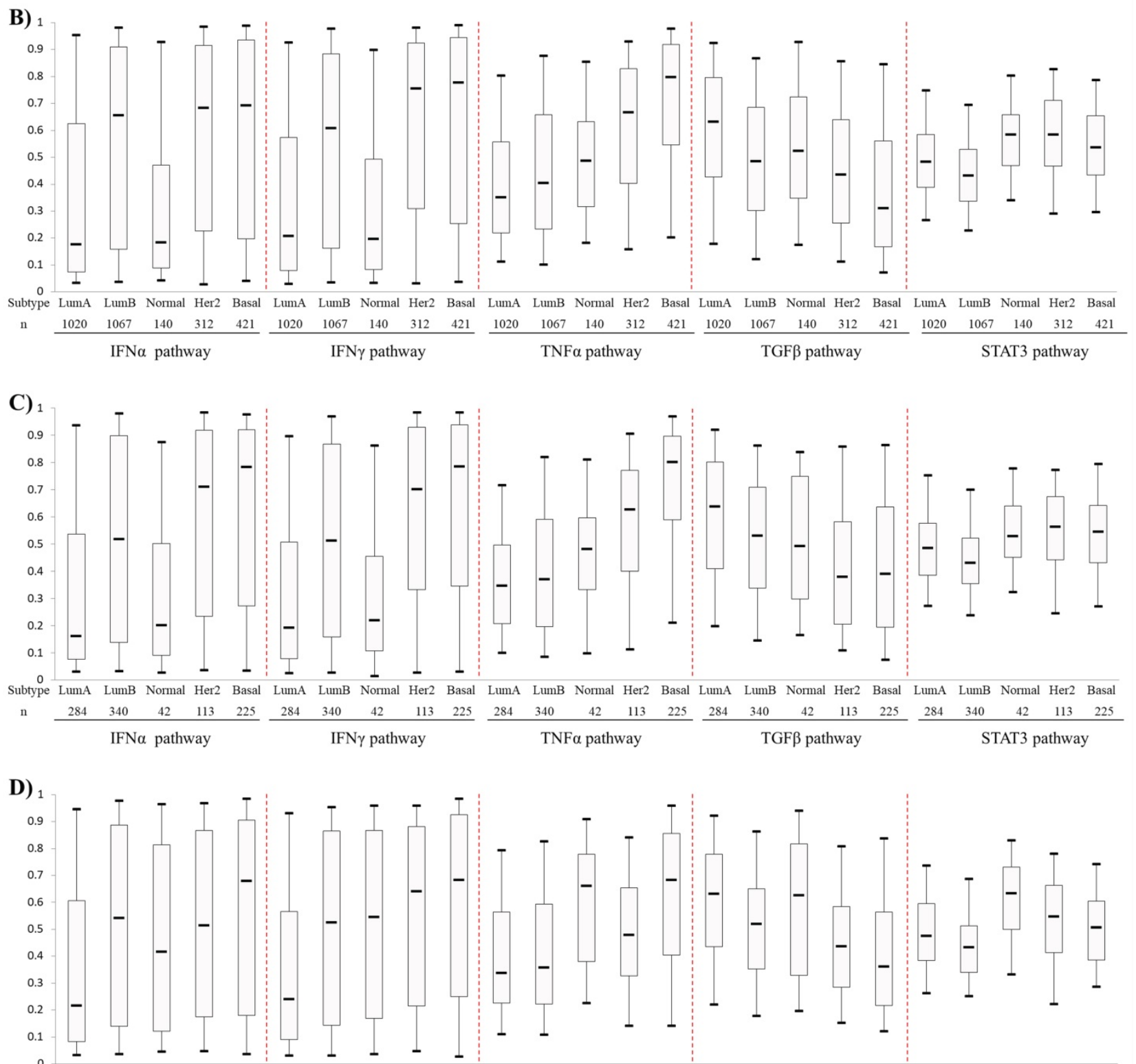

Subtype LumA LumB Normal Her2 Basal LumA LumB Normal Her2 Basal LumA LumB Normal Her2 Basal LumA LumB Normal Her2 Basal LumA LumB Normal Her2 Basal

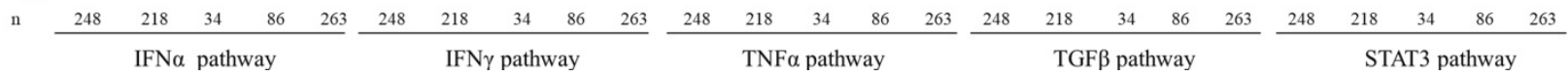

Figure 1. Comparison of IFNa, IFNy, TNFa, TGF $\beta$ and STAT3 pathway activities among PAM50-based intrinsic subtypes of breast cancers. A) Patient cohort 1. B) Cohort 2. C) Cohort 3. D) Cohort 4. The four BC cohorts were merged from 42 Affymetrix microarray datasets as described in Materials and methods. Box-Whisker plots were used to show pathway activity, and the five statistics (5th, 25th, 50th, 75th and 95th percentile) were represented by the lower whisker, the lower box part, the solid line, the upper box part and the upper whisker, respectively. Notably, three datasets (GSE16446, GSE25055 and GSE25065) were present in both Cohort 1 and Cohort 4. To avoid repeated counting of sample data here, these three datasets were removed from cohort 1 when calculating pathway activity. Lum A: luminal A; Lum B: luminal B; Normal: Normal-like; Her2: HER2-enriched; Basal: basal-like. 


\section{IFN $\alpha$, IFNy, TNF $\alpha$ and TGF $\beta$ signaling are associated with recurrence risk of BLBC}

Associations of the five immunity-related pathways with BC recurrence risk were first examined using univariate Cox regression (Fig. 2). When all BC samples were tested or just luminal $A$, luminal B, Normal-like BC subtypes were tested, none of the five pathways were prognostic for $B C$ recurrence. By contrast, when basal-like subtype was tested in Cox regression, the IFNY (overall HR 0.77, 95\% CI 0.69-0.86, $p=4.26 \mathrm{E}-06$, Fig. 2B) and TNFa (overall HR 0.83, 95\% CI 0.74-0.92, $p=5.96 \mathrm{E}-04$, Fig. 2C) pathways were significant favorable factors while the TGF $\beta$ pathway (overall HR 1.33, 95\% CI 1.2-1.48, $p$ $=1.85 \mathrm{E}-07$, Fig. 2D) was significant unfavorable factors for disease-free survival. The IFNa pathway was also a prognostic factor (overall HR $0.85,95 \%$ CI 0.76-0.95, p = 4.95E-03, Fig. 2A) but had weaker effect than the IFN $\gamma$ and TNFa pathways. Notably, higher IFN $\gamma$ and TNFa pathway activity were also associated with lower recurrence risk in HER2-enriched BC. No association between the STAT3 pathway and BC recurrence was observed in any of $\mathrm{BC}$ subtypes (Supplementary Fig. S2).

Multivariate regression analysis revealed that the association of IFNa, IFN $\gamma$, TNFa and TGF $\beta$ pathways with recurrence risk remain significant in BLBC after adjusting for different clinical variables including age, tumor grade and size, and status of lymph node, estrogen receptor (ER), progesterone receptor (PR) and HER2 in the three cohorts (Supplementary Fig. S3).

The impact of the IFNa, IFN $\gamma$, TNFa and TGF $\beta$ pathways on BLBC recurrence was further supported by Kaplan-Meier analysis. As shown in Fig. S4, HER2-enriched and basal-like BCs have worst prognosis among the five PAM50 subtypes in the 3 cohorts. Based on IFN $\gamma$, TNFa or TGF $\beta$ pathway activities, BLBC could be further stratified into 3 subgroups with significantly different recurrence risks (Fig. 3). Similar to the results in Cox regression analysis, subgroups of BLBC with higher IFNY (log-rank $p=7.45 \mathrm{E}-04,0.02$ and 0.03 respectively) and TNFa (log-rank $p=0.06,0.06$ and 6.31E-03 respectively) activity had significantly lower recurrence risk in all $3 \mathrm{BC}$ cohorts (Fig. 3 ). Recurrence risk of BLBC was also low in subgroups with high IFNa (log-rank $p=0.51,0.11$ and 0.22 respectively) or low TGF $\beta$ (log-rank $p=2.73 \mathrm{E}-05,0.14$ and 0.20 respectively) pathway activities, which, however, was not as significant as that for IFN $\gamma$ and TNFa pathways (Fig. 3).

\section{The combined application of TNF $\alpha$ and TGF $\beta$ pathway activities improves the prediction of BLBC recurrence}

It is well-known that IFNa, IFN $\gamma$ and $\mathrm{TNFa}$ signaling mediate antitumor immunity whereas TGF $\beta$ signaling induces tumor immunosuppression [37-42]. This is consistent with our results here that IFNa, IFN $\gamma$ and TNFa pathways are favorable survival factors, while TGF $\beta$ is an unfavorable survival factor in BLBC. We wondered whether the prediction of BC recurrence could be further improved by using a combination of an immunosuppressive pathway and an immune activation pathway. Pathway combinations were tested in all PAM50 subtypes, and Cox regression analysis showed that synergistic effects of pathway combination on recurrence prediction were only observed in BLBC and HER2enriched BC (Fig. 4A). In BLBC, the combination of TGF $\beta$ pathway with either IFNa, IFN $\gamma$ or TNFa pathways archived more significant overall $\mathrm{HR}(\mathrm{HR}=$ $0.73,0.69,0.70$, and $\mathrm{p}=5.95 \mathrm{E}-08,4.92 \mathrm{E}-11,1.04 \mathrm{E}-09$ for the 3 combinations, respectively) with respect to the association with cancer recurrence than the 4 individual pathways $(\mathrm{HR}=1.23,0.85,0.77,0.83$, and $p$ $=1.85 \mathrm{E}-07,4.95 \mathrm{E}-03,4.26 \mathrm{E}-06,5.96 \mathrm{E}-04$, respectively) (Fig. 4A). Moreover, the synergistic effects of these 3 combinations in recurrence prediction for BLBC were observed in all the 3 individual cohorts (Fig. 4B and Fig. 2). By contrast, although combination of TGF $\beta$ pathway with IFNa or IFN $\gamma$ also archived more significant overall HR than individual pathways in HER2-enriched BC (Fig. 4A), we found the combination effects were only present in cohort 2 but not in the other 2 cohorts (Fig. 4C, Fig. 2A and B).

The synergistic effects of pathway combinations in recurrence prediction for BLBC were also evaluated using Kaplan-Meier analysis. The difference of recurrence risk among the 3 subgroups stratified based on the TGF $\beta$ \& TNFa combination score (Supplementary Fig. S5A-C) was more significant than those stratified based on only TGF $\beta$ or TNFa pathway activity alone (Fig. 3G-L) in all the 3 cohorts. When BLBCs were stratified into 2 subgroups, the recurrence risks were much more different among subgroups stratified based on TGF $\beta$ \& TNFa combination score than those based on TGF $\beta$ or TNFa pathway alone (log-rank $p=2.81 \mathrm{E}-05,0.03$ and $4.06 \mathrm{E}-04$ for combination vs. $p=4.98 \mathrm{E}-05,0.36$ and 0.29 for TGF $\beta$ alone and $p=0.15,0.18$ and 0.48 for TNFa alone) in the 3 cohorts (Fig. 5). By contrast, the synergistic effect of TGF $\beta$ pathway combined with IFNa or IFN $\gamma$ pathway was only observed in BLBCs from cohorts 3 and 4, regardless of whether the samples were stratified into three subgroups 
(Supplementary Fig. S5D-L, Fig. 3A-F) or two
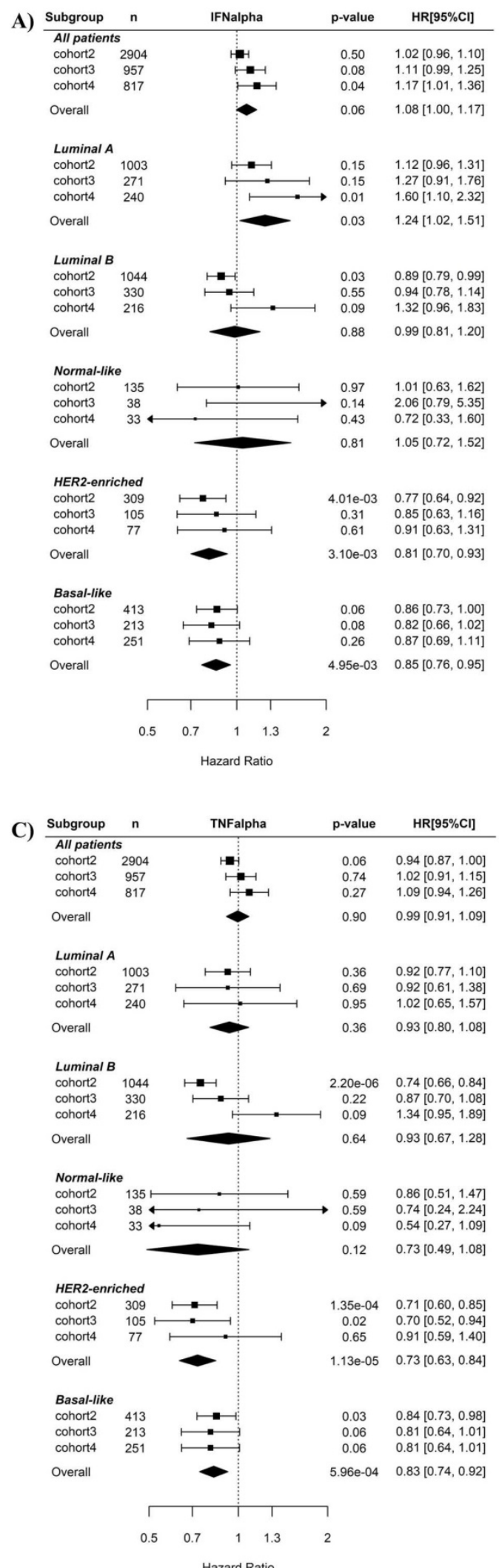

subgroups (Supplementary Fig. S6).
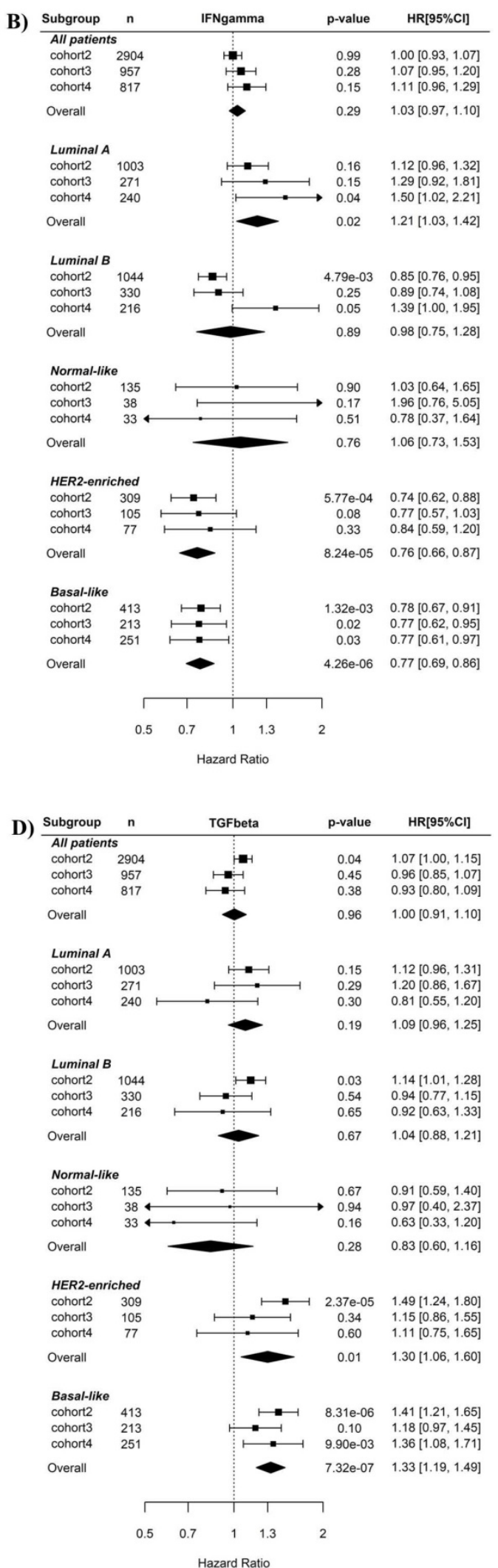

Figure 2. Cox regression analysis of the associations of the IFNa, IFNy, TNF $\alpha$ and TGF $\beta$ pathways with recurrence risk in different subtypes of breast cancers. A) IFNa pathway. B) IFNy pathway. C) TNFa pathway. D) TGF $\beta$ pathway. The three BC cohorts annotated with patient's survival information were analyzed here. The pathway activities were used as continuous variables. The recurrence risk with the increase of the pathway activity was indicated by HR (presented per one-SD increment) as shown in forest plot. The overall effect of HR was calculated using a random-effects model, and the significance of the overall effects across multiple cohorts was estimated by $\mathrm{Z}$ test. HRs are shown in forest plots, in which the squares and horizontal lines represent the $\mathrm{HR}$ and $95 \% \mathrm{Cl}$ for the individual variables, while the diamonds represent the $\mathrm{HR}$ and $95 \% \mathrm{Cl}$ for the overall estimate. IFNa: IFNa; IFNg: IFNy; TNFa: TNFa; TGFb: TGF $\beta$. 

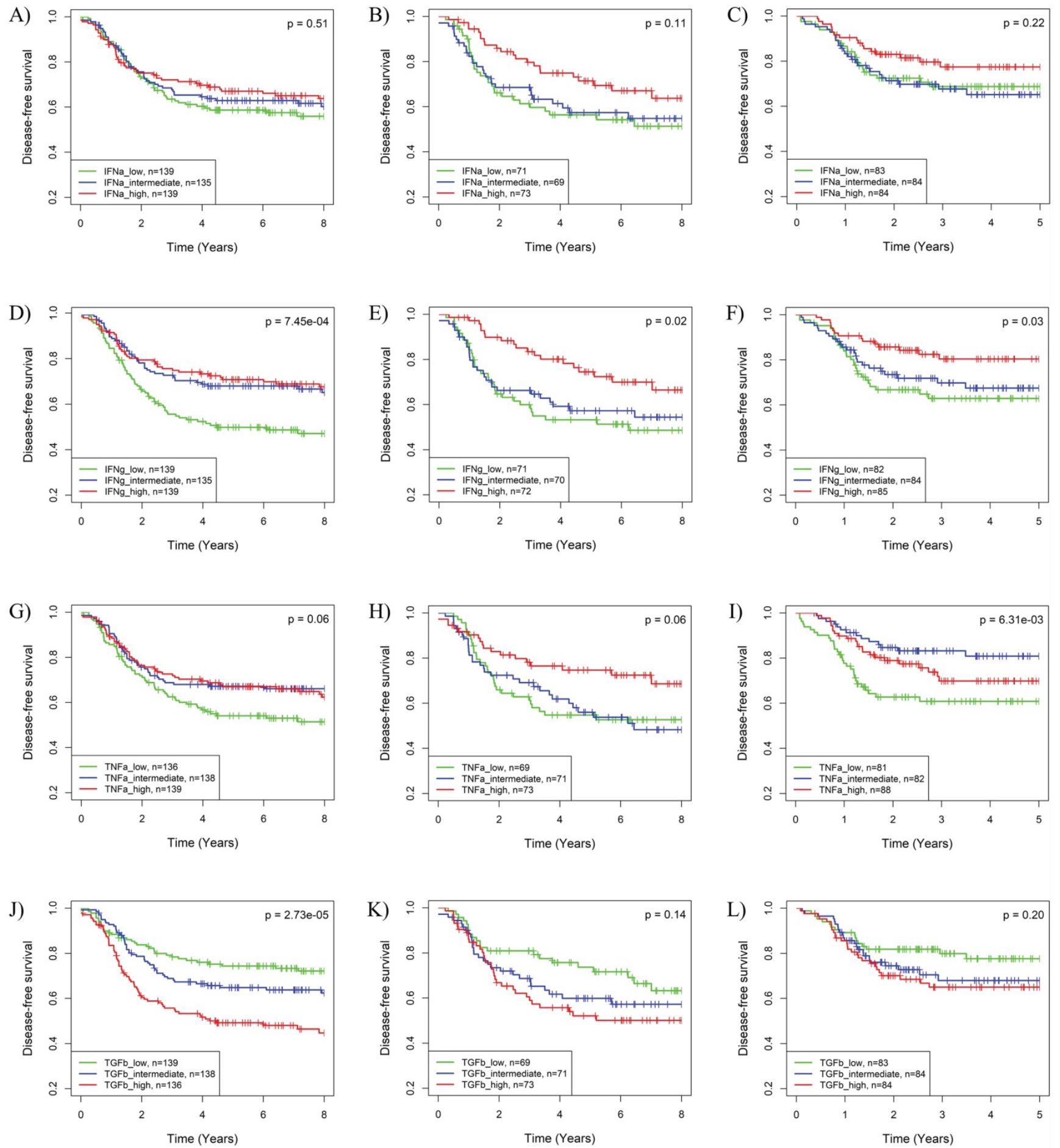

Figure 3. Kaplan-Meier analysis of the associations of the IFNa, IFNy, TNFa and TGF $\beta$ pathway activities with recurrence risk of BLBC. The three BC cohorts annotated with patient's survival information were analyzed, including cohort 2 (A, D, G, J), cohort 3 (B, E, H, K) and cohort 4 (C, F, I, L). The BLBCs were stratified into three subgroups based on tertile splits of predicted activities of the IFNa (A-C), IFNy (D-F), TNFa (G-I) or TGF $(\mathrm{J}-\mathrm{L})$ pathway (high: $>2 / 3$ percentile; low $\leq 1 / 3$ percentile; intermediate: $\leq 2 / 3$ percentile and $>1 / 3$ percentile) for each cohort, and Kaplan-Meier analysis was performed to compare the probabilities of DFS among each of the three subgroups. IFNa: IFNa; IFNg: IFNY; TNFa: TNFa; TGFb: TGF $\beta$.

\section{Combined use of TNF $\alpha$ and TGF $\beta$ pathway signatures improve recurrence prediction for BLBC treated with adjuvant chemotherapy}

Chemotherapy is the main method for treating BLBC. We asked whether these phenomena observed above are the effects of these immune-related pathway signaling on response of $\mathrm{BC}$ cells to chemotherapy. Analysis of BCs in cohort 1 revealed that basal-like and HER2-enriched BCs had best response to neoadjuvant chemotherapy among the 5 subtypes (Supplementary Fig. S7A). Cox regression analysis on BLBC in this cohort showed that none of the 4 pathways (IFNa, IFNץ, TNFa and TGF $\beta$ ) were 
associated with neoadjuvant response (Supplementary Fig. S7B). Combined application of TNFa and TGF $\beta$ signatures moderately enhanced the correlation with neoadjuvant therapy responses but did not reach statistical significance $(p=0.11)$ (Supplementary Fig. S7B).

We also examined the impact of the above 4 pathways on the recurrence risk of BLBC treated with adjuvant chemotherapy. Only Cohorts 2 and 4 were used in this analysis because only 21 basal-like samples in Cohort 3 had chemotherapy information annotated. Cox regression analysis revealed that only TGF $\beta$ pathway was significantly associated with recurrence of BLBC after chemotherapy treatment (Supplementary Fig. S7C). Combination of TGF $\beta$ with IFNY or TNFa strongly enhanced this association in cohort 2 (Supplementary Fig. S7C). In Kaplan-Meier analysis, however, TGF $\beta$ and TNFa pathway combination exhibited synergistic effects on enhancing the association in both cohorts (log-rank $p$ $=1.09 \mathrm{E}-03,2.56 \mathrm{E}-02$ for combination $v s . p=0.13,0.63$ for TNFa and $p=1.33 \mathrm{E}-02,3.15 \mathrm{E}-02$ for TGF $\beta$ alone and alone) (Supplementary Fig. S8). In contrast, in Kaplan-Meier analysis, such synergistic effects were observed only in cohort 2 for TGF $\beta$-IFN $\gamma$ pathway combination (Supplementary Fig. S9).
A)

\begin{tabular}{l} 
Signature \\
\hline TGFb \\
IFNa \\
IFNa \& TGFb \\
IFNg
\end{tabular}

B)

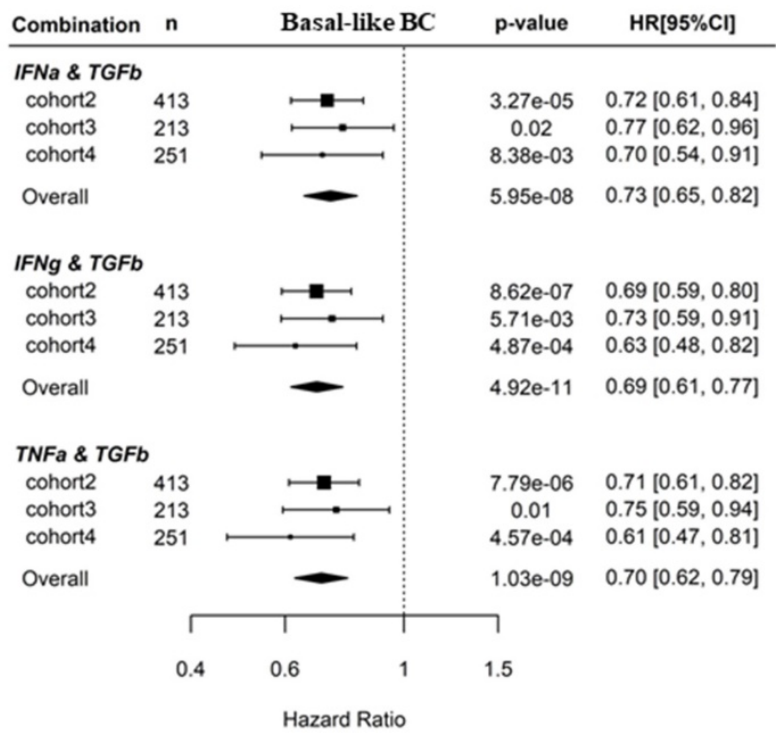

C)

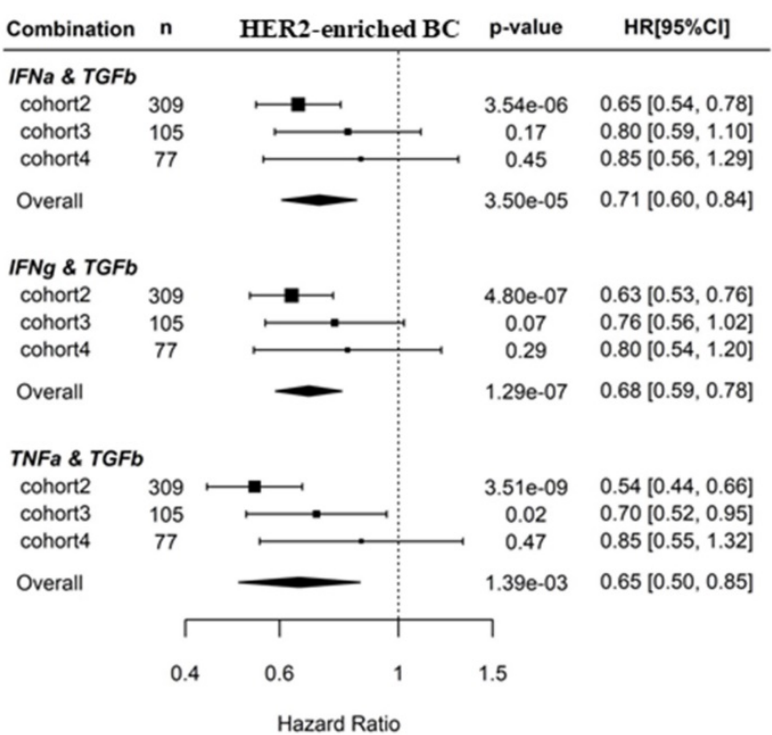

Figure 4. Pathway combinations improve the prediction of BLBC prognosis. A). Comparison of prediction efficiency of various pathway combinations in five PAM50 subtypes BC. Combination of the TGF $\beta$ pathway with the IFNa, IFNY or TNFa pathways was tested here as indicated. The overall HR for recurrence risk shown in forest plot was based on Cox regression analysis of three breast cancer cohorts as described in Methods. Scores for pathway combinations, as described in Methods, were used as continuous variable in Cox regression analysis and the $\mathrm{HR}[95 \% \mathrm{Cl}]$ is presented per one-SD increment. B) Forest plot showing the prediction efficiency of pathway combinations for BLBC in three individual cohorts. C) Forest plot showing the prediction efficiency of pathway combinations for HER2-enriched BC in three individual cohorts. 

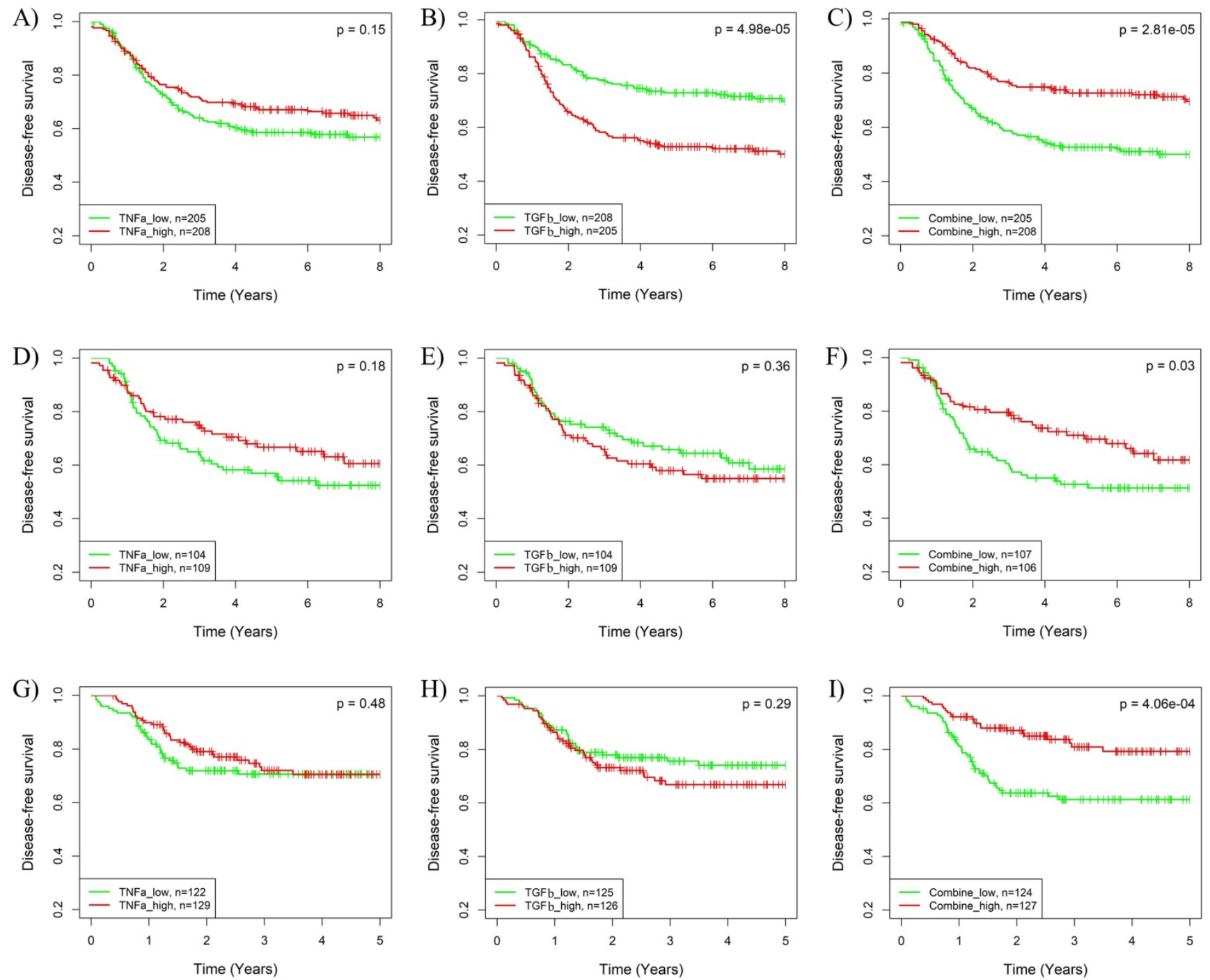

Figure 5. Kaplan-Meier analysis of the synergistic effect of TNFa and TGF $\beta$ pathway in prognosis prediction of BLBCs. Three BC cohorts with survival information were analyzed, including cohort 2 (A-C), cohort $3(D-F)$ and cohort 4 (G-I). The patients were stratified into two groups based on median splits of predicted pathway activities or pathway combination scores in each cohort. A, D, G) Stratification based on TNFa pathway activity. B, E, H) Stratification based on TGF $\beta$ pathway activity. C, F, I) Stratification based on the combination scores of TNFa and TGFß pathway. TNFa: TNFa; TGFb: TGF $\beta$.

\section{Activated memory CD4 T cells are enriched in BLBC with high TNF $\alpha$ and low TGF $\beta$ pathway activity}

Besides affecting anti-cancer immunity, TNFa and TGF $\beta$ signaling also have direct tumorsuppressing or oncogenic effects on tumor cells. To uncover the potential molecular mechanisms that could explain the synergistic effects of TNFa/TGF $\beta$ pathway combination in prognostic prediction of BLBC, we first examined the associations of TNFa and TGF $\beta$ signaling with the alterations of six cancer-related pathways, including p53, BRAF, EGFR, androgen receptor (AR), beta-catenin (BCAT) and PI3K pathways that were reported to play important roles in the molecular pathogenesis of BLBC $[43,44]$. The BLBC were stratified into 4 subgroups in each cohort based on median values of the TNFa and TGF $\beta$ pathway activities, i.e., high-TNFa/low-TGF $\beta$ (TNFa+TGF $\beta$-), low-TNFa/high-TGF $\beta$ (TNFa$\mathrm{TGF} \beta+)$, high-TNFa/high-TGF $\beta$ (TNFa+TGF $\beta+)$ and low-TNFa/low-TGF $\beta$ (TNFa-TGF $\beta$-). We first compared the activities of the above-mentioned 6 pathways among these 4 subgroups. Enrichment of some of the pathways was associated with either TNFa or TGF $\beta$ signaling, such as p53 signaling enriched in TNFa- BC and EGFR signaling enriched in TGF $\beta+$ BC (Fig. 6). However, none of the 6 pathways showed specific enrichment or suppression in $\mathrm{TNF} \alpha+\mathrm{TGF} \beta$ - or TNFa-TGF $\beta+$ subgroups of BLBC (Fig. 6).

Considering that the TNFa and TGF $\beta$ signaling strength in cancer cells reflects the TNFa and TGF $\beta$ levels in intercellular matrix, we ask whether intracellular TNFa and TGF $\beta$ signaling is associated with infiltration of any specific type of immune cells 
in BLBC. CIBERSORT [36] was used here to calculate the fractions of 22 immune cell subsets in BLBC. Among these 22 cell subsets, infiltrations of 4 subsets, including activated memory CD4 T (Tm) cells and resting (M0), pro-inflammatory (M1) and anti-inflammatory (M2) macrophages, were found to correlate with TNFa or TGF $\beta$ signaling in BLBC (Fig. 6, Supplementary Fig. S10). Higher TNFa signaling in cancer cells was associated with higher activated Tm cell level and lower M2/higher M1 macrophage levels, while higher TGF $\beta$ signaling was associated with higher M0 macrophages level (Fig. 6). However, only activated Tm cells in these 4 cell subsets showed specific enrichment in BLBCs with TNFa+TGF $\beta$ - or
TNFa-TGF $\beta+$ (Fig. 6). Activated Tm cell infiltration was mainly observed in $\mathrm{TNFa}^{+}$particularly $\mathrm{TNFa}+\mathrm{TGF} \beta-\mathrm{BLBC}$ (Fig. 6), and this trend was more clearly presented when using a scatter plot to visualize the corresponding data shown in the heatmap (supplementary Fig. S11). TNFa-TGF $\beta$ BLBC had higher level of activated Tm subset than TNFa-TGF $\beta+$ BLBC $(p=0.04$, Mann-Whitney U Test), while $\mathrm{TNFa}+\mathrm{TGF} \beta+$ BLBC had higher level of activated Tm cells than TNFa-TGF $\beta$ - BLBC $(p<1 \mathrm{E}-04)$ and TNFa+TGF $\beta-$ BLBC had higher level than $\mathrm{TNF} \alpha+\mathrm{TGF} \beta+\mathrm{BLBC}(p=1 \mathrm{E}-04)$, suggesting that both TNFa and TGF $\beta$ signaling affect the infiltration of activated Tm cells in BLBC (supplementary Fig. S11).
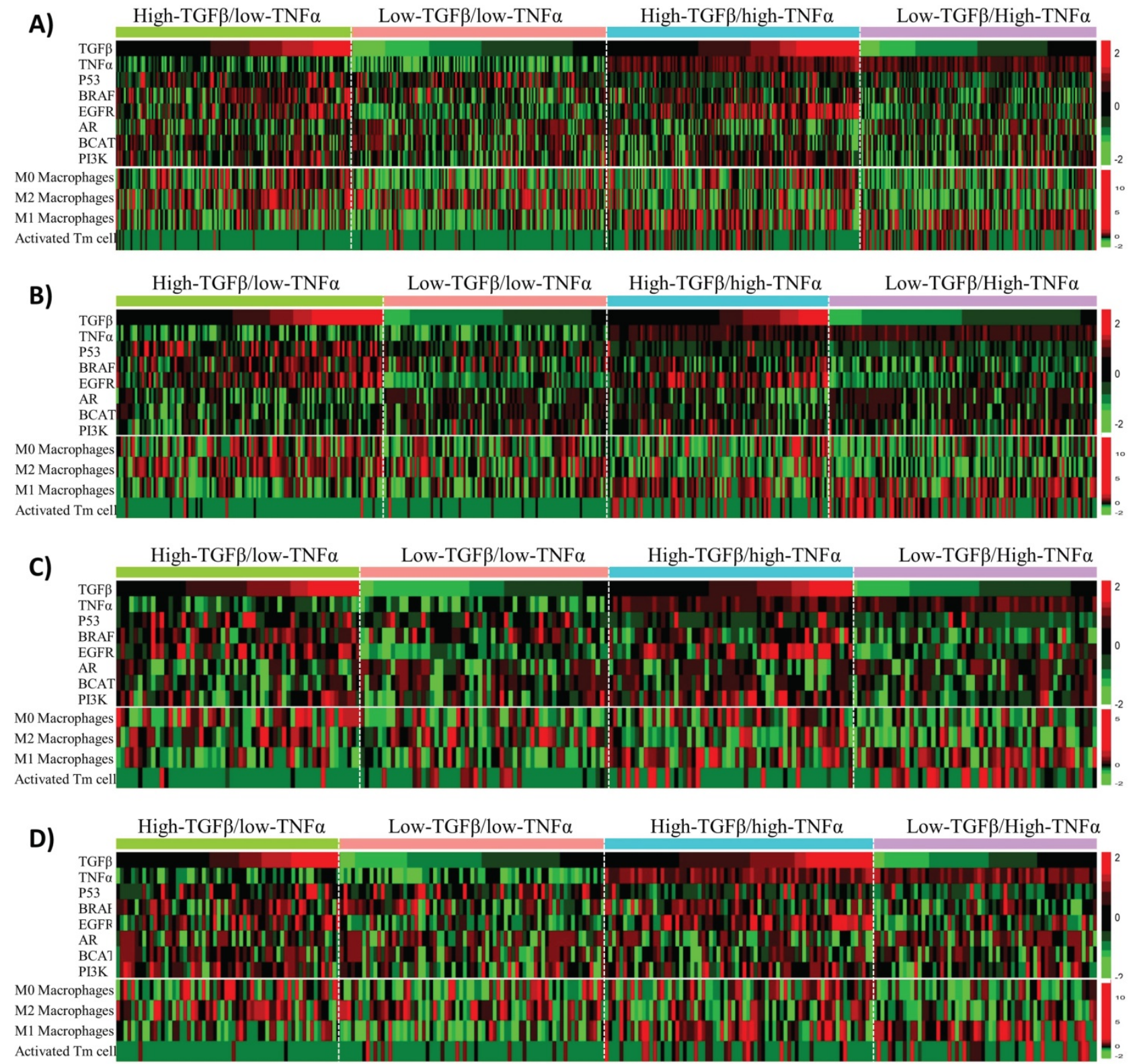

Figure 6. Associations of TNF $\alpha$ and TGF $\beta$ pathway status with activities of six BLBC-related pathways and infiltrations of four immune cell subsets in BLBC. BLBC from four BC patient cohorts were tested. A) Cohort 1. B) Cohort 2. C) Cohort 3. D) Cohort 4. Four BLBC subgroups, including high-TNFa/low-TGF $\beta$, low-TNFa/high-TGF $\beta$, high-TNFa/high-TGF $\beta$ and low-TNFa/low-TGF $\beta$ as indicated, were stratified from each cohort based on median values of the TNFa and TGF $\beta$ pathway activities, the heatmap was used to depict relative pathway activities and immune cell subset levels across the four BLBC subgroups. Each row represents one pathway or an immune cell subset as indicated. Each column represents one BLBC sample. 


\section{Discussion}

At present, few clinical variables show predictive ability for prognosis of BLBC. Hence, we tried to identify genomic predictors of prognosis in patients with BLBCs. In the present study, we use four large independent breast cancer cohorts to examine the association of five immunity-related pathways with breast cancer prognosis. Analysis of these cohorts showed that among the five PAM50 subtypes of BC, BLBC and HER2-enriched BC were most sensitive to neoadjuvant chemotherapy and have worst prognosis. These results are well consistent with previous reports [45, 46], suggesting the reliability of gene expression data of these cohorts. Notably, four immunity-related pathways, including the IFNa, IFNY, TNFa and TGF $\beta$ pathways, are associated with BLBC recurrence rate in varying degrees. While the TGF $\beta$ pathway is an unfavorable prognostic factor, the other three are favorable. Combined use of TNFa and TGF $\beta$ pathway activities improve prediction of recurrence risk of BLBC.

Here, we demonstrate that the combination of $\mathrm{TNFa} / \mathrm{TGF} \beta$ or $\mathrm{IFN} \gamma / \mathrm{TGF} \beta$ pathways can predict BLBC prognosis and activated CD4+ $\mathrm{T}$ cell levels. More importantly, TNFa/TGF $\beta$ combination has better synergistic effects in prognosis prediction than IFN $\gamma / T G F \beta$ combination. In BC, particularly in the aggressive triple-negative/basal-like subgroup, patient outcome is closely associated with the immune infiltration of tumors. While infiltration of cytotoxic $\mathrm{T}$ cells and assisting T-helper 1 cells are associated with good prognosis, infiltration of tumor-associated macrophages (TAMs) is linked to poor prognosis [47]. Since interferon is the key cytokine to activate cytotoxic $\mathrm{T}$ cells and assist $\mathrm{T}$ helper cells, while TGF- $\beta$ activates TAMs $[48,49]$, it is not surprising that interferon and TGF- $\beta$ signaling are respectively a favorable and an unfavorable factor for BLBC prognosis in this study. TNF-a signaling also positively affects immune activation in the tumor microenvironment through a positive feedback mechanism between TNFa levels and M1 macrophage activation [50]. In fact, the data here also show that higher TNFa signaling is associated with higher M1 and lower M2 macrophage levels (Fig. 6). Although the association of IFNY signaling as a single variable with cancer recurrence was more significant than that of TNFa signaling (Fig. 2 and 3), the combined effects of TNFa/TGF $\beta$ signaling were more significant than combination of IFN $\gamma /$ TGF $\beta$ signaling in BLBC (Fig. 4 and 5). Interestingly, we observed that TNFa signaling strength increased gradually from luminal A, luminal B, Normal-like, HER2-enriched and basal-like subtypes, whereas the TGF $\beta$ signaling exhibited an exact opposite pattern (Fig. 1). Probably, TNFa and TGF $\beta$ signaling are more complementary than IFN $\gamma$ and TGF $\beta$ signaling in immune regulation, which may provide a potential explanation for better synergistic effects of $\mathrm{TNFa} / \mathrm{TGF} \beta$ combination in prediction of BLBC prognosis. Besides potential application in clinical outcome prediction, these findings may also help to understand the molecular pathogenesis of BLBCs.

Memory $\mathrm{T}$ cells are largely resting cells that become active after antigen stimulation. Analysis of $T$ cell subpopulations in circulation, lymph nodes, and tumor sites in tumor-bearing mice revealed severe loss of $\mathrm{T}$ memory cells during tumor progression, indicating a continuous competition between cancer and T memory cells [51]. Evaluation of the leukocyte composition in breast tumors indicated that activated T-memory cells are effector memory cells and a gene signature for this cell subset is associated with lower cancer recurrence $[52,53]$. It has been proposed that these tumor-specific memory cells can survive and maintain effective immunosurveillance with the function of long-term detection and removal of residual tumor cells [52]. In addition, several studies reported that TNFa and TGF $\beta$ signaling are involved in the generation and activation of memory $\mathrm{T}$ cell populations [54-56]. These data, together with our findings that BLBCs with low TGF $\beta$ and high TNFa pathway activities was enriched with activated Tm cells and has better prognosis, further support that cooperation of TNFa and TGF $\beta$ signaling may play an important anti-tumor role in BLBC through mechanisms at least including generation and activation of memory CD4 T cells.

There are currently no rigorously validated methods to guide the prognosis of BLBC patients. In fact, the data we provide here show that it is possible to develop such a test. Future studies will focus on extending these findings in more retrospective cohort of BLBC patients, and finally in a prospective-based clinical trial aimed at protecting low-risk BLBC patients from harmful and redundant adjuvant chemotherapy.

\section{Supplementary Material}

Supplementary figures and tables. http://www.ijbs.com/v17p0670s1.pdf

\section{Acknowledgements}

This work was supported in part by NIH-NIMHD U54MD007598, U54CA143931, NIH/NCI1U54CA14393， U56 CA101599-01; Department-of-Defense Breast Cancer Research Program grant BC043180, NIH/NCATS CTSI UL1TR000124 to J.V. Vadgama; Accelerating 
Excellence in Translational Science (AXIS) Pilot Grants G0812D05, NIH/NCI SC1CA200517 and 9 SC1 GM135050-05 to Y. Wu.

\section{Competing Interests}

Liu has equity interest in Bluewater Biotech LLC.

\section{References}

1. Perou CM, Sorlie T, Eisen MB, van de Rijn M, Jeffrey SS, Rees CA, et al. Molecular portraits of human breast tumours. Nature. 2000; 406: 747-52.

2. Sorlie T, Perou CM, Tibshirani R, Aas T, Geisler S, Johnsen H, et al. Gene expression patterns of breast carcinomas distinguish tumor subclasses with clinical implications. Proc Natl Acad Sci U S A. 2001; 98: 10869-74.

3. Sorlie T, Tibshirani R, Parker J, Hastie T, Marron JS, Nobel A, et al. Repeated observation of breast tumor subtypes in independent gene expression data sets. Proc Natl Acad Sci U S A. 2003; 100: 8418-23.

4. Slamon DJ, Leyland-Jones B, Shak S, Fuchs H, Paton V, Bajamonde A, et al. Use of chemotherapy plus a monoclonal antibody against HER2 for metastatic breast cancer that overexpresses HER2. N Engl J Med. 2001; 344: 783-92.

5. Livasy CA, Karaca G, Nanda R, Tretiakova MS, Olopade OI, Moore DT, et al. Phenotypic evaluation of the basal-like subtype of invasive breast carcinoma. Mod Pathol. 2006; 19: 264-71.

6. Rodriguez-Pinilla SM, Sarrio D, Honrado E, Hardisson D, Calero F, Benitez J, et al. Prognostic significance of basal-like phenotype and fascin expression in node-negative invasive breast carcinomas. Clin Cancer Res. 2006; 12: 1533-9.

7. Paik S, Shak S, Tang G, Kim C, Baker J, Cronin M, et al. A multigene assay to predict recurrence of tamoxifen-treated, node-negative breast cancer. N Engl J Med. 2004; 351: 2817-26.

8. Wang Y, Klijn JG, Zhang Y, Sieuwerts AM, Look MP, Yang F, et al. Gene-expression profiles to predict distant metastasis of lymph-node-negative primary breast cancer. Lancet. $2005 ; 365: 671-9$.

9. Sotiriou C, Wirapati P, Loi S, Harris A, Fox S, Smeds J, et al. Gene expression profiling in breast cancer: understanding the molecular basis of histologic grade to improve prognosis. J Natl Cancer Inst. 2006; 98: 262-72.

10. Chang HY, Nuyten DS, Sneddon JB, Hastie T, Tibshirani R, Sorlie T, et al Robustness, scalability, and integration of a wound-response gene expression signature in predicting breast cancer survival. Proc Natl Acad Sci U S A. 2005; 102: 3738-43.

11. van 't Veer LJ, Dai H, van de Vijver MJ, He YD, Hart AA, Mao M, et al. Gene expression profiling predicts clinical outcome of breast cancer. Nature. 2002; 415: 530-6.

12. Hallett RM, Dvorkin A, Gabardo CM, Hassell JA. An algorithm to discover gene signatures with predictive potential. J Exp Clin Cancer Res. 2010; 29: 120.

13. Hallett RM, Hassell JA. E2F1 and KIAA0191 expression predicts breast cancer patient survival. BMC Res Notes. 2011; 4: 95

14. Nielsen TO, Hsu FD, Jensen $\mathrm{K}$, Cheang $\mathrm{M}$, Karaca G, Hu Z, et al. Immunohistochemical and clinical characterization of the basal-like subtype of invasive breast carcinoma. Clin Cancer Res. 2004; 10: 5367-74.

15. Carey LA, Perou CM, Livasy CA, Dressler LG, Cowan D, Conway K, et al. Race, breast cancer subtypes, and survival in the Carolina Breast Cancer Study. JAMA. 2006; 295: 2492-502.

16. Abd El-Rehim DM, Pinder SE, Paish CE, Bell J, Blamey RW, Robertson JF, et al. Expression of luminal and basal cytokeratins in human breast carcinoma. J Pathol. 2004; 203: 661-71.

17. Cheang MC, Voduc D, Bajdik C, Leung S, McKinney S, Chia SK, et al. Basal-like breast cancer defined by five biomarkers has superior prognostic value than triple-negative phenotype. Clin Cancer Res. 2008; 14: 1368-76.

18. Mulligan AM, Pinnaduwage D, Bull SB, O'Malley FP, Andrulis IL. Prognostic effect of basal-like breast cancers is time dependent: evidence from tissue microarray studies on a lymph node-negative cohort. Clin Cancer Res. 2008; 14: $4168-74$.

19. Dent $\mathrm{R}$, Trudeau M, Pritchard KI, Hanna WM, Kahn HK, Sawka CA, et al. Triple-negative breast cancer: clinical features and patterns of recurrence. Clin Cancer Res. 2007; 13: 4429-34

20. Blows FM, Driver KE, Schmidt MK, Broeks A, van Leeuwen FE, Wesseling I, et al. Subtyping of breast cancer by immunohistochemistry to investigate a relationship between subtype and short and long term survival: a collaborative analysis of data for 10,159 cases from 12 studies. PLoS Med. 2010; 7: e1000279.

21. Wirapati P, Sotiriou C, Kunkel S, Farmer P, Pradervand S, Haibe-Kains B, et al. Meta-analysis of gene expression profiles in breast cancer: toward a unified understanding of breast cancer subtyping and prognosis signatures. Breast Cancer Res. 2008; 10: R65.

22. Desmedt C, Haibe-Kains B, Wirapati P, Buyse M, Larsimont D, Bontempi G, et al. Biological processes associated with breast cancer clinical outcome depend on the molecular subtypes. Clin Cancer Res. 2008; 14: 5158-65.

23. Yau C, Esserman L, Moore DH, Waldman F, Sninsky J, Benz CC. A multigene predictor of metastatic outcome in early stage hormone receptor-negative and triple-negative breast cancer. Breast Cancer Res. 2010; 12: R85.
24. Sabatier R, Finetti P, Mamessier E, Raynaud S, Cervera N, Lambaudie E, et al. Kinome expression profiling and prognosis of basal breast cancers. Mol Cancer. 2011; 10: 86

25. Teschendorff AE, Miremadi A, Pinder SE, Ellis IO, Caldas C. An immune response gene expression module identifies a good prognosis subtype in estrogen receptor negative breast cancer. Genome Biol. 2007; 8: R157.

26. Kreike B, van Kouwenhove M, Horlings $H$, Weigelt B, Peterse H, Bartelink H, et al. Gene expression profiling and histopathological characterization of triple-negative/basal-like breast carcinomas. Breast Cancer Res. 2007; 9: R65.

27. Rody A, Holtrich U, Pusztai L, Liedtke C, Gaetje R, Ruckhaeberle E, et al. $\mathrm{T}$-cell metagene predicts a favorable prognosis in estrogen receptor-negative and HER2-positive breast cancers. Breast Cancer Res. 2009; 11: R15.

28. Liu D. Concomitant dysregulation of the estrogen receptor and BRAF/MEK signaling pathways is common in colorectal cancer and predicts a worse prognosis. Cell Oncol (Dordr). 2019; 42: 197-209.

29. Liu D. AR pathway activity correlates with AR expression in a HER2-dependent manner and serves as a better prognostic factor in breast cancer. Cell Oncol (Dordr). 2020; 43: 321-33.

30. Liu $\mathrm{D}, \mathrm{Wu} \mathrm{Y}$. Association of an anaplastic lymphoma kinase pathway signature with cell de-differentiation, neoadjuvant chemotherapy response, and recurrence risk in breast cancer. Cancer Commun (Lond). 2020; 40: 422-34.

31. Liu D. Identification of a prognostic LncRNA signature for ER-positive, ER-negative and triple-negative breast cancers. Breast Cancer Res Treat. 2020; 183: 95-105.

32. Bild AH, Yao G, Chang JT, Wang Q, Potti A, Chasse D, et al. Oncogenic pathway signatures in human cancers as a guide to targeted therapies. Nature. 2006; 439: 353-7.

33. Gatza ML, Lucas JE, Barry WT, Kim JW, Wang O, Crawford MD, et al. A pathway-based classification of human breast cancer. Proc Natl Acad Sci U S A. 2010; 107: 6994-9.

34. Liu D, Liu X, Xing M. Activities of multiple cancer-related pathways are associated with BRAF mutation and predict the resistance to BRAF/MEK inhibitors in melanoma cells. Cell Cycle. 2014; 13: 208-19.

35. Gendoo DM, Ratanasirigulchai N, Schroder MS, Pare L, Parker JS, Prat A, et al. Genefu: an R/Bioconductor package for computation of gene expression-based signatures in breast cancer. Bioinformatics. 2016; 32: 1097-9.

36. Newman AM, Liu CL, Green MR, Gentles AJ, Feng W, Xu Y, et al. Robust enumeration of cell subsets from tissue expression profiles. Nat Methods. 2015; $12: 453-7$

37. Shou $\mathrm{P}$, Chen $\mathrm{Q}$, Jiang J, Xu C, Zhang J, Zheng C, et al. Type I interferons exert anti-tumor effect via reversing immunosuppression mediated by mesenchymal stromal cells. Oncogene. 2016; 35: 5953-62.

38. Whitman E, Barber A. NKG2D receptor activation of NF-kappaB enhances inflammatory cytokine production in murine effector CD8(+) T cells. Mol Immunol. 2015; 63: 268-78.

39. Wong JL, Muthuswamy R, Bartlett DL, Kalinski P. IL-18-based combinatorial adjuvants promote the intranodal production of CCL19 by NK cells and dendritic cells of cancer patients. Oncoimmunology. 2013; 2: e26245.

40. Yang Z, Qi Y, Lai N, Zhang J, Chen Z, Liu M, et al. Notch1 signaling in melanoma cells promoted tumor-induced immunosuppression via upregulation of TGF-beta1. J Exp Clin Cancer Res. 2018; 37: 1.

41. Haque S, Morris JC. Transforming growth factor-beta: A therapeutic target for cancer. Hum Vaccin Immunother. 2017; 13: 1741-50.

42. Batlle E, Massague J. Transforming Growth Factor-beta Signaling in Immunity and Cancer. Immunity. 2019; 50: 924-40.

43. Gupta GK, Collier AL, Lee D, Hoefer RA, Zheleva V, Siewertsz van Reesema LL, et al. Perspectives on Triple-Negative Breast Cancer: Current Treatment Strategies, Unmet Needs, and Potential Targets for Future Therapies. Cancers (Basel). 2020; 12

44. Anders CK, Abramson V, Tan T, Dent R. The Evolution of Triple-Negative Breast Cancer: From Biology to Novel Therapeutics. Am Soc Clin Oncol Educ Book. 2016; 35: 34-42.

45. Prat A, Pineda E, Adamo B, Galvan P, Fernandez A, Gaba L, et al. Clinical implications of the intrinsic molecular subtypes of breast cancer. Breast. 2015; 24 Suppl 2: S26-35.

46. Haibe-Kains B, Desmedt C, Loi S, Culhane AC, Bontempi G, Quackenbush J, et al. A three-gene model to robustly identify breast cancer molecular subtypes. J Natl Cancer Inst. 2012; 104: 311-25.

47. Savas P, Salgado R, Denkert C, Sotiriou C, Darcy PK, Smyth MJ, et al. Clinical relevance of host immunity in breast cancer: from TILs to the clinic. Nat Rev Clin Oncol. 2016; 13: 228-41.

48. Provance OK, Lewis-Wambi J. Deciphering the role of interferon alpha signaling and microenvironment crosstalk in inflammatory breast cancer. Breast Cancer Res. 2019; 21: 59.

49. Williams CB, Yeh ES, Soloff AC. Tumor-associated macrophages: unwitting accomplices in breast cancer malignancy. NPJ Breast Cancer. 2016; 2

50. Hao NB, Lu MH, Fan YH, Cao YL, Zhang ZR, Yang SM. Macrophages in tumor microenvironments and the progression of tumors. Clin Dev Immunol. 2012; 2012: 948098.

51. Bhattacharyya S, Md Sakib Hossain D, Mohanty S, Sankar Sen G, Chattopadhyay S, Banerjee S, et al. Curcumin reverses T cell-mediated adaptive immune dysfunctions in tumor-bearing hosts. Cell Mol Immunol. 2010; 7: 306-15. 
52. Gu-Trantien C, Loi S, Garaud S, Equeter C, Libin M, de Wind A, et al. CD4(+) follicular helper $\mathrm{T}$ cell infiltration predicts breast cancer survival. J Clin Invest. 2013; 123: 2873-92.

53. Ruffell B, Au A, Rugo HS, Esserman LJ, Hwang ES, Coussens LM. Leukocyte composition of human breast cancer. Proc Natl Acad Sci U S A. 2012; 109: 2796-801.

54. Agius E, Lacy KE, Vukmanovic-Stejic M, Jagger AL, Papageorgiou AP, Hall S, et al. Decreased TNF-alpha synthesis by macrophages restricts cutaneous immunosurveillance by memory CD4+ T cells during aging. J Exp Med. 2009; 206: $1929-40$

55. Croft M. The TNF family in T cell differentiation and function--unanswered questions and future directions. Semin Immunol. 2014; 26: 183-90.

56. Nguyen TP, Sieg SF. TGF-beta inhibits IL-7-induced proliferation in memory but not naive human CD4(+) T cells. J Leukoc Biol. 2017; 102: 499-506. 